\title{
Radiative Phi decays at KLOE
}

\author{
The KLOE Collaboration * \\ Presented by Paolo Gauzzi ${ }^{\dagger}$ \\ Dipartimento di Fisica, Università degli Studi di Roma "la Sapienza” e INFN Sezione di Roma, \\ P.le A.Moro 2, 00185 Rome (Italy) \\ E-mail: Paolo.Gauzzi@roma1.infn.it
}

We describe the status of the analyses in progress on light meson spectroscopy in the KLOE experiment. We present the analyses of $\phi$ decays into $f_{0}(980) \gamma$ and $a_{0}(980) \gamma$, the study of the Dalitz plot of the $\eta \rightarrow \pi^{+} \pi^{-} \pi^{0}$ and $\eta \rightarrow 3 \pi^{0}$ decays, the measurement of the branching ratio of $\eta \rightarrow \pi^{0} \gamma \gamma$, and the measurement of the ratio $\operatorname{Br}\left(\phi \rightarrow \eta^{\prime} \gamma\right) / \operatorname{Br}(\phi \rightarrow \eta \gamma)$. All the presented results are based on a sample of about $450 \mathrm{pb}^{-1}$ of integrated luminosity collected during the 2001-2002 data taking. From the 2004-2005 data taking a factor 5 improvement in the integrated luminosity is expected.

International Europhysics Conference on High Energy Physics

July 21st - 27th 2005

Lisboa, Portugal

${ }^{*}$ The KLOE Collaboration: F. Ambrosino, A. Antonelli, M. Antonelli, C. Bacci, P. Beltrame, G. Bencivenni, S. Bertolucci, C. Bini, C. Bloise, V. Bocci, F. Bossi, D. Bowring, P. Branchini, R. Caloi, P. Campana, G. Capon, T. Capussela, F. Ceradini, S. Chi, G. Chiefari, P. Ciambrone, S. Conetti, E. De Lucia, P. De Simone, G. De Zorzi, S. Dell'Agnello, A. Denig, A. Di Domenico, C. Di Donato, S. Di Falco, B. Di Micco, A. Doria, M. Dreucci, G. Felici, A. Ferrari, M. L. Ferrer, G. Finocchiaro, C. Forti, P. Franzini, C. Gatti, P. Gauzzi, S. Giovannella, E. Gorini, E. Graziani, M. Incagli, W. Kluge, V. Kulikov, F. Lacava, G. Lanfranchi, J. Lee-Franzini, D. Leone, M. Martini, P. Massarotti, W. Mei, L. Merola, S. Miscetti, M. Moulson, S. Müller, F. Murtas, M. Napolitano, F. Nguyen, M. Palutan, E. Pasqualucci, A. Passeri, V. Patera, F. Perfetto, L. Pontecorvo, M. Primavera, P. Santangelo, E. Santovetti, G. Saracino, B. Sciascia, A. Sciubba, F. Scuri, I. Sfiligoi, T. Spadaro, M. Testa, L. Tortora, P. Valente, B. Valeriani, G. Venanzoni, S. Veneziano,

A. Ventura, R. Versaci, G. Xu

†speaker. 
The KLOE detector operates at the Frascati $e^{+} e^{-}$collider DAФNE, that works at the peak of the $\phi(1020)$ resonance. The results presented here are based on data collected in the years 2001 and 2002 for an integrated luminosity of $\sim 450 \mathrm{pb}^{-1}$.

The scalar mesons $f_{0}(980)$ and $a_{0}(980)\left(\mathrm{J}^{P C}=0^{++}\right)$are not easily interpreted as ordinary $\mathrm{q} \overline{\mathrm{q}}$ mesons, belonging to the ${ }^{3} P_{0}$ nonet; alternative hypotheses have been proposed: $q \bar{q} q \bar{q}$ states or $K \overline{\mathrm{K}}$ bound states. The branching ratios of $\phi \rightarrow f_{0} \gamma$ and $\phi \rightarrow a_{0} \gamma$, as well as the $f_{0}$ and $a_{0}$ mass shapes, are sensitive to the structure of these particles. KLOE has already published analyses of $\eta \pi^{0} \gamma$ and of $\pi^{0} \pi^{0} \gamma$ final states, based on a data sample of about $17 \mathrm{pb}^{-1}$ collected in year 2000[1].

The $f_{0}(980)$ is searched for in $\pi^{0} \pi^{0} \gamma$ and $\pi^{+} \pi^{-} \gamma$ final states. $e^{+} e^{-} \rightarrow \pi^{0} \pi^{0} \gamma$ is selected by looking for fully neutral events with 5 energy deposits in the calorimeter. In fig.1(Left) the Dalitz plot for the selected sample of $1.5 \times 10^{5}$ events is reported. The two sharp bands correspond to $e^{+} e^{-} \rightarrow \omega\left(\rightarrow \pi^{0} \gamma\right) \pi^{0}$, while the structure close to the high $M_{\pi \pi}^{2}$ corner is due to $\phi \rightarrow f_{0}(\rightarrow$ $\left.\pi^{0} \pi^{0}\right) \gamma$. A fit to the Dalitz plot of different models to extract the $f_{0}$ parameters and all the relevant contribution to the $\pi^{0} \pi^{0} \gamma$ final state, is in progress.
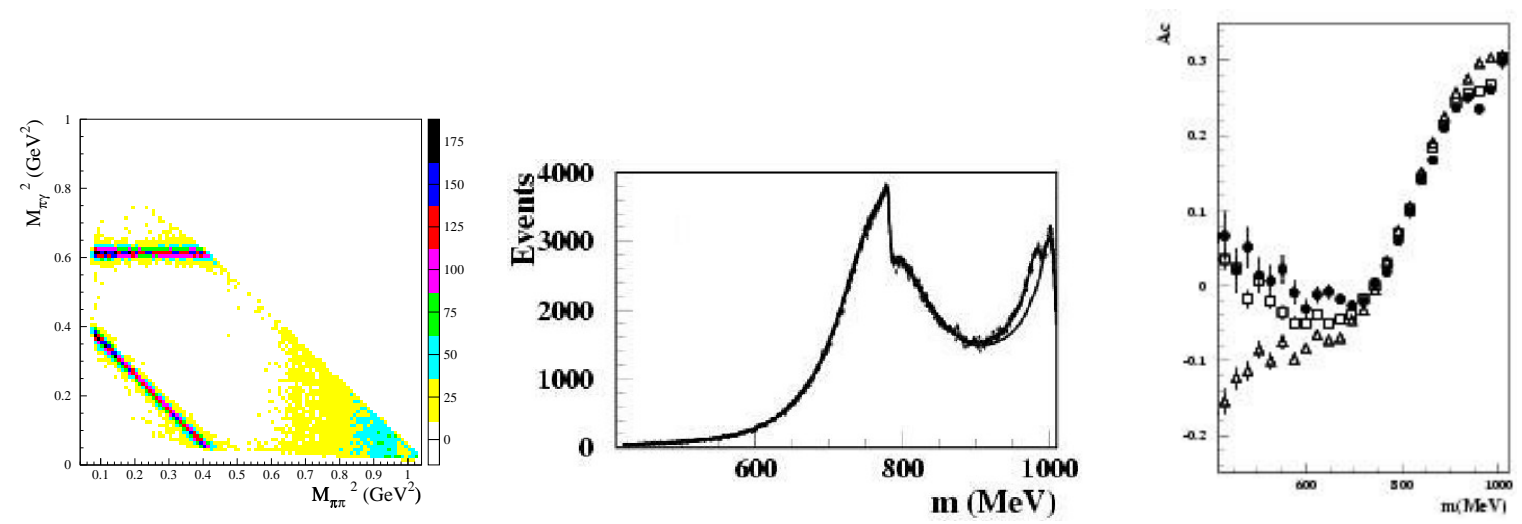

Figure 1: (Left) Dalitz plot for $e^{+} e^{-} \rightarrow \pi^{0} \pi^{0} \gamma$ events. (Center) $\mathrm{M}_{\pi \pi}$ spectrum for $e^{+} e^{-} \rightarrow \pi^{+} \pi^{-} \gamma$ events. (Right) Charge asymmetry $\left(A=\left[N^{+}\left(\theta>90^{\circ}\right)-N^{+}\left(\theta<90^{\circ}\right)\right] /\left[N^{+}\left(\theta>90^{\circ}\right)+N^{+}\left(\theta<90^{\circ}\right)\right]\right)$ : data (circles) compared to $\mathrm{MC}$ without (triangles) and with (squares) $f_{0}$ contribution.

The search for $\phi \rightarrow f_{0}\left(\rightarrow \pi^{+} \pi^{-}\right) \gamma$ is characterized by the presence of high rate irreducible backgrounds due to the initial state radiation (ISR), and to $e^{+} e^{-} \rightarrow \pi^{+} \pi^{-} \gamma$, occuring either through the $\phi$ or through the $\rho^{0}$ tail (final state radiation, FSR). A further lower rate background is due to the decay chain $\phi \rightarrow \rho^{ \pm}\left(\rightarrow \pi^{ \pm} \gamma\right) \pi^{ \pm}$. Since the ISR cross-section is peaked at small photon angles respect to the beam line, the $f_{0}$ events are searched for in the large angle region $45^{\circ}<\theta<135^{\circ}$. Fig.1(Center) shows the spectrum obtained at $\sqrt{s}=M_{\phi}$; the bump appearing around $980 \mathrm{MeV}$ is the $f_{0}$ signal. Due to the C-odd term coming from the interference between FSR and ISR, a charge asymmetry is present in the two pion state (see fig.1(Right)). The deviation from the Monte Carlo (MC) prediction, based only on ISR and FSR, is an independent evidence of the $f_{0}$ signal. By adding, following ref.[2], to the $\mathrm{MC}$ simulation an $f_{0}$ amplitude, the data-MC agreement is recovered.

The $a_{0}(980)$ is searched for in $e^{+} e^{-} \rightarrow \eta \pi^{0} \gamma$ final states, selected through the decays $\eta \rightarrow$ $\gamma \gamma$ and $\eta \rightarrow \pi^{+} \pi^{-} \pi^{0}$. In the first case the final state is fully neutral with 5 photons detected in the calorimeter, while in the second case also two charged tracks are present. About 23000 
events have been selected for the first decay chain, and about 5000 events for the second one. The preliminary branching ratio obtained from the two channels are: $\operatorname{Br}\left(\phi \rightarrow \eta \pi^{0} \gamma\right)=(7.25 \pm 0.15) \times$ $10^{-5}$ from $\eta \rightarrow \gamma \gamma$ and $\operatorname{Br}\left(\phi \rightarrow \eta \pi^{0} \gamma\right)=(7.45 \pm 0.19) \times 10^{-5}$ from $\eta \rightarrow \pi^{+} \pi^{-} \pi^{0}$. In fig. 2 , the two $\eta \pi^{0}$ invariant mass spectra are reported. Also in this case a fit to several models, in order to extract the relavant $a_{0}(980)$ parameters, is in progress.
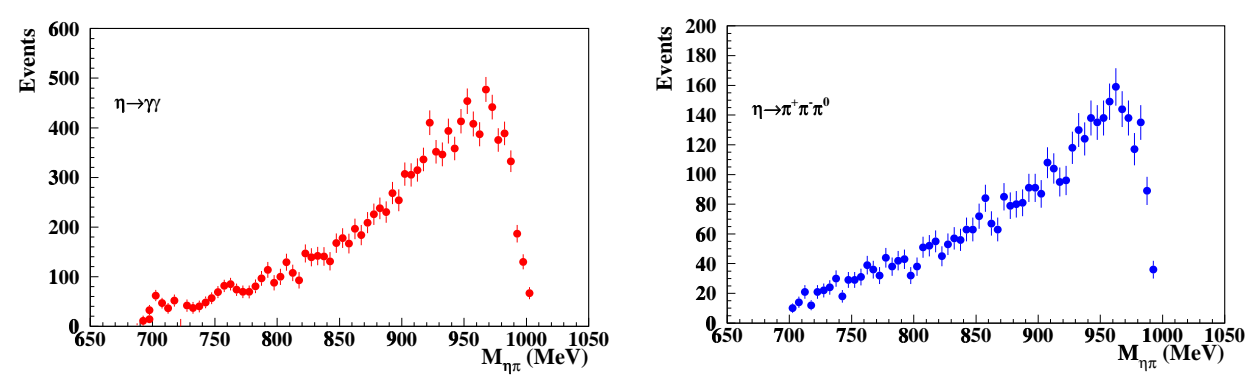

Figure 2: $\eta \pi^{0}$ invariant mass spectra for the $\eta \rightarrow \gamma \gamma$ (left) and $\eta \rightarrow \pi^{+} \pi^{-} \pi^{0}$ (right) samples.

About 18 millions of $\eta$ mesons have been produced at DAФNE during 2001 and 2002 data taking. This large sample has been used by KLOE to study both the charged and the fully neutral decays of $\eta \rightarrow 3 \pi . \quad \eta \rightarrow \pi^{+} \pi^{-} \pi^{0}$ proceeds via isospin breaking and and its decay amplitude at lowest order chiral perturbation theory (ChPT) is: $A(s, t, u)=\frac{1}{Q^{2}} \frac{m_{K}^{2}}{m_{\pi}^{2}}\left(m_{\pi}^{2}-m_{K}^{2}\right) \frac{M(s, t, u)}{3 \sqrt{3} F_{0}^{2}}$ where $Q^{2} \equiv\left(m_{s}^{2}-\hat{m}^{2}\right) /\left(m_{d}^{2}-m_{u}^{2}\right)$ and $\hat{m}=\frac{1}{2}\left(m_{u}+m_{d}\right)$. Thus the decay $\eta \rightarrow \pi^{+} \pi^{-} \pi^{0}$ can be used in order to determine the quantity $Q^{2}$. In fig.3(Left) the Dalitz plot for more than 1.2 millions of events is shown. The variables $\mathrm{X}$ and $\mathrm{Y}$ are defined as: $X=\sqrt{3} \frac{T_{+}-T_{-}}{Q_{\eta}}$ and $Y=\frac{3 T_{0}}{Q_{\eta}}-1$ where $Q_{\eta}=m_{\eta}-$ $2 m_{\pi^{+}}-m_{\pi^{0}}$ and $T_{+}, T_{-}, T_{0}$ are the kinetic energies of the pions. The measured distribution has been fitted to $|A(X, Y)|^{2}=\left(1+a Y+b Y^{2}+c X+d X^{2}+e X Y+f Y^{3}\right)$ (see tab.1). As expected from $\mathrm{C}$ conservation the $c$ and $e$ parameters are compatible with zero; moreover a sizeable cubic term in $Y$ has to be taken into account. From this result, the value $Q=22.8 \pm 0.4$ has been obtained[3], that seems to imply a violation of the Dashen theorem.
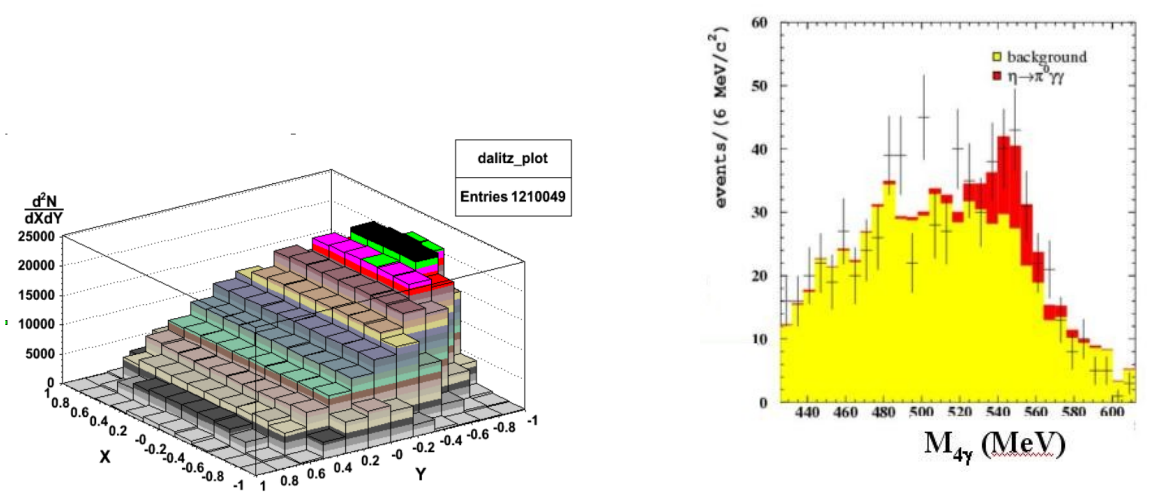

Figure 3: (Left) The observed Dalitz plot distribution in $\eta \rightarrow \pi^{+} \pi^{-} \pi^{0}$ events. (Right) 4 photon invariant mass spectrum for $\eta \rightarrow \pi^{0} \gamma \gamma$.

The Dalitz plot density for $\eta \rightarrow \pi^{0} \pi^{0} \pi^{0}$ can be expressed in terms of only one parameter $\alpha$, $|A(Z)|^{2} \simeq 1+2 \alpha Z$ where $Z=\frac{2}{3} \sum_{i=1}^{3}\left(\frac{3 E_{i}-m_{\eta}}{m_{\eta}-3 m_{\pi}}\right)^{2}$. A fit has been done on a high purity (98.5\%) 
Table 1: Results of the fit to the $\eta \rightarrow \pi^{+} \pi^{-} \pi^{0}$ Dalitz plot density.

\begin{tabular}{|c|c|c|c|c|c|c|}
\hline $\mathrm{P}\left(\chi^{2}\right)$ & $\mathrm{a}$ & $\mathrm{b}$ & $\mathrm{c}$ & $\mathrm{d}$ & $\mathrm{e}$ & $\mathrm{f}$ \\
\hline $60 \%$ & $-1.072 \pm 0.006$ & $0.117 \pm 0.006$ & $0.0001 \pm 0.0029$ & $0.047 \pm 0.006$ & $-0.006 \pm 0.008$ & $0.13 \pm 0.01$ \\
& \pm 0.007 & \pm 0.006 & ${ }_{-0.0021}^{+0.0003}$ & \pm 0.005 & ${ }_{-0.0}^{+0.013}$ & \pm 0.02 \\
\hline
\end{tabular}

sample and the resulting parameter is $\alpha=-0.013 \pm 0.005_{\text {stat }} \pm 0.004_{\text {syst }}$, that can be compared to the best current measurement $\alpha=-0.031 \pm 0.004[4]$.

The $\eta \rightarrow \pi^{0} \gamma \gamma$ decay is a test of the $\mathrm{O}\left(p^{6}\right)$ ChPT predictions, since in this case the $\mathrm{O}\left(p^{2}\right)$ and $\mathrm{O}\left(p^{4}\right)$ terms give very small contributions. KLOE has performed a measurement of the branching ratio by selecting events with only 5 photons in the final state. The main background processes are: $\phi \rightarrow f_{0} \gamma \rightarrow \pi^{0} \pi^{0} \gamma, \phi \rightarrow a_{0} \gamma \rightarrow \eta \pi^{0} \gamma, e^{+} e^{-} \rightarrow \omega \pi^{0} \rightarrow \pi^{0} \pi^{0} \gamma$, and $\phi \rightarrow \eta \gamma \rightarrow \pi^{0} \pi^{0} \pi^{0} \gamma$ (wrongly recontructed as 5 photon). In fig.3(Right) the invariant mass of the four less energetic photons is reported. The KLOE preliminary result, $\operatorname{Br}\left(\eta \rightarrow \pi^{0} \gamma \gamma\right)=(8.0 \pm 2.7 \pm 1.4) \times 10^{-5}$, is not in agreement with the previuos measurements[5], while is in agreement with the predictions based on $\mathrm{ChPT} \mathrm{O}\left(\mathrm{p}^{6}\right)$ calculations.

The number of $\eta^{\prime}$ mesons produced in 2001-2002 data taking is nearly 100000. KLOE has anlyzed the decay chains $\phi \rightarrow \eta^{\prime} \gamma ; \eta^{\prime} \rightarrow \pi^{+} \pi^{-} \eta ; \eta \rightarrow \pi^{0} \pi^{0} \pi^{0}$ and $\phi \rightarrow \eta^{\prime} \gamma ; \eta^{\prime} \rightarrow \pi^{0} \pi^{0} \eta$; $\eta \rightarrow \pi^{+} \pi^{-} \pi^{0}$, in order to measure the ratio $R=\Gamma\left(\phi \rightarrow \eta^{\prime} \gamma\right) / \Gamma(\phi \rightarrow \eta \gamma)$, that is related to the pseudoscalar mixing angle in the flavor basis and to the $\eta^{\prime}$ gluonic content. The final state is charachterized by two charged pions and seven photons, and has no background with the same topology in KLOE. After background subtraction we observe $3405 \pm 61 \pm 28 \phi \rightarrow \eta^{\prime} \gamma$ events (fig.4). We normalize to the number of observed $\eta \rightarrow \pi^{0} \pi^{0} \pi^{0}$ decays to obtain a preliminary measurement of $R=\left(4.76 \pm 0.08_{\text {stat }} \pm 0.20_{\text {syst }}\right) \times 10^{-3}$, where the systematic error is dominated by the knowledge of the intermediate BR's of the $\eta^{\prime}$. This result is in agreement with our previous estimate I61. based on a different final state.
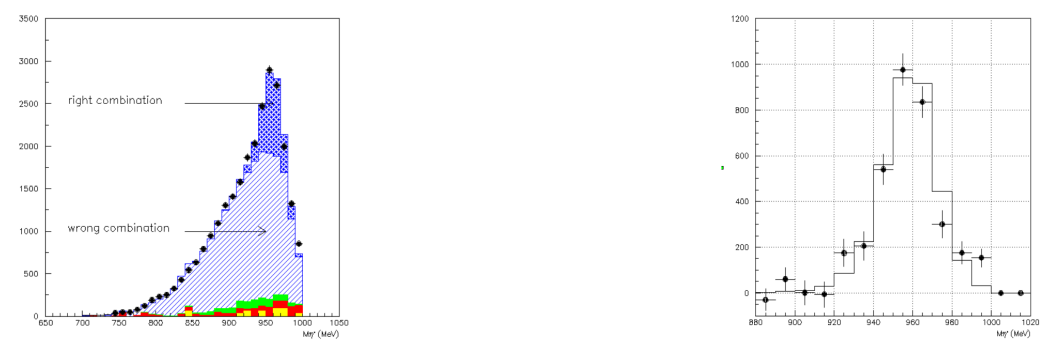

Figure 4: Six photons and two tracks invariant mass: (Left) all combinations; (Right) $\eta^{\prime}$ mass spectrum after combinatorial background subtraction.

\section{References}

[1] KLOE coll.,A. Aloisio et al., Phys.Lett.B536(2002)209; Phys.Lett.B537(2002)21.

[2] H.Czyz, A.Grzelinska and J.H.Kühn, hep-ph/0412239.

[3] B.V.Martemyanov and V.S.Sopov, Phys.Rev.D71(2005)017501.

[4] W.B.Tippens et al., Phys.Rev.Lett.87(2001)192001.

[5] Alde et al., Z.Phys.C25(1984)225; N. Knecht et al., Phys.Lett.B589(2004)14.

[6] KLOE coll., A. Aloisio et al., Phys.Lett.B541 (2002)45. 